\title{
Spatially selective binaural hearing aids
}

\section{Luca Brayda}

RBCS dept., Istituto Italiano di

Tecnologia, Genoa, Italy

luca.brayda@iit.it

\section{Federico Traverso}

DITEN - University of Genoa,

Genoa, Italy

federico.traverso@ginevra.dibe.unige.it

\section{Luca Giuliani}

RBCS dept., Istituto Italiano di

Tecnologia, Genoa, Italy

luca.giuliani@iit.it

Francesco Diotalevi

RBCS dept., Istituto Italiano di

Tecnologia, Genoa, Italy

francesco.diotalevi@iit.it

Permission to make digital or hard copies of all or part of this work for personal or classroom use is granted without fee provided that copies are not made or distributed for profit or commercial advantage and that cot ma this notice and the full citation on the first page. Copyrights for components of this work owned by others than ACM must be honis for components of this work owned by others than ACM must be honored. Abstracting with credit is permitted. To copy otherwise, or republish, to post on servers or to redistribute to lists, requires prior specific

permission and/or a fee. Request permissions from Permissions@acm.or UbiComp/ISWC'15 Adjunct, September 7-11, 2015, Osaka, Japan. (c) 2015 ACM 978-1-4503-3575-1/15/09...\$15.00.

http://dx.doi.org/10.1145/2800835.2806207

\section{Stefania Repetto}

Sinear s.r.l, Genoa, Italy

\section{Sara Sansalone}

Andrea Trucco

Genoa, Italy

Giulio Sandini

RBCS dept., Istituto Italiano di

Tecnologia, Genoa, Italy

giulio.sandini@iit.it

\begin{abstract}
Traditional hearing aids are limited by the absence of spatial selectivity. Superdirective microphone array can recover such limit, performing a spatial filtering to achieve an augmented SNR. We present Glassense, a platform hosting a double microphone array connected to a processing unit and mounted on the frame of common glasses. The platform has the potential of delivering binaural spatially selective audio inputs, allowing ecological pointing of acoustic sources through head motion. The designed microphone arrays exhibit a gain suitable to improve the speech reception threshold of hearing-impaired subjects, obtained on a lightweight and scalable hardware setup
\end{abstract}

\section{Author Keywords}

Hearing aid; disability; microphone arrays;

superdirective beamforming; real time

\section{ACM Classification Keywords}

H.5.m. Information interfaces and presentation (e.g., HCI): Miscellaneous; K.4.2. Computer and Society:

Social issues

\section{Introduction}

The preservation of spatial information of sound sources is paramount for maintaining speech intelligibility, especially when hearing loss occurs [1]. 
Binaural hearing devices outperform monaural hearing aid devices, as they help to preserve essential spatial cues such as interaural time difference (ITD) and interaural level difference (ILD) [2]. As most of literature proves [3], binaural hearing is preferable compared to especially for localization and listening in noise. These tasks heavily rely on such spatial cues. The dynamics of head motion dominates such cues [4]. However, although visual cues help to point to acoustic source of interest, hearing-impaired individuals are less efficient in doing so [5]. Instead of using only one microphone per ear, binaural multi-microphne hearing aids have shown to be promising solutions, because the effect of unwanted spatial sound sources can be decreased [6]. In fact, recent works involving subjects with hearing loss reported a clear preference for directional over omnidirectional settings [7]; directional hearing aids are also preferred when noise is present and the signal of interest is in front of, and relatively near to, the listener [8]. Directional processing seems not to require significant additional listening effort, although the optimal amount of directionality seems to be task-dependent [9]. However, evaluation of setups based on microphone arrays as hearing aids is often confined to simulating real world conditions [7], therefore few wearable prototypes exist [10] which allow testing the hearing aids in ecological and ergonomic setups, i.e. with constraints on weight and accounting for wearability and usability. The aim of this work is to build a wearable multichannel binaural hearing aid, which hosts an effective beamforming algorithm able to improve speech intelligibility. The system, called Glassense, can be potentially incorporated in a pair of glasses as a wearable assistive device.

\section{Measuring effectiveness of hearing aids}

Hearing aids are designed to increase the perceived Signal-to-Noise Ratio (SNR). The necessary gain for an aid to be effective, expressed in $\mathrm{dB}$, must allow an impaired individual to reach his/her Speech Reception Threshold (SRT), defined as the SNR at which half of a set of utterances are well understood [11]. A hearing aid must gain between 5 and $15 \mathrm{~dB}$ to let the SRT of an impaired match that of a normal individual [12].

\section{Microphone arrays as hearing aids}

A microphone array can be used to feed traditional hearing aids to add spatial filtering of the acoustic field. A measure of the spatial filtering performance is given by the directivity index (DI), the value of the array gain versus isotropic noise (i.e., noise coming from all directions with equal intensity) with respect to a single omnidirectional sensor. The DI computed versus frequency represents the ability of the array to achieve a SNR level over a desired frequency band. A weighted sum of $\mathrm{DI}(f)$ allows to obtain a broadband intelligibilityweighted directivity as follows:

$D_{I W}=\sum_{i}^{I} \gamma_{i} D I\left(f_{i}\right)$

where $D I\left(f_{i}\right)$ is the array gain at frequency $f_{i}$ and $\gamma_{i}$ is a weight indicating the importance of $f_{i}$ in human speech perception, [3]. Here, frequency range is expressed as a discrete set of one-thirds of octaves band between $300 \mathrm{~Hz}$ and $5000 \mathrm{~Hz}$ and $f_{i}$ is the center frequency of the i-th band. The goal of an arraybased hearing aid is to achieve a $D I\left(f_{i}\right)$ so that $D_{I W}$ 


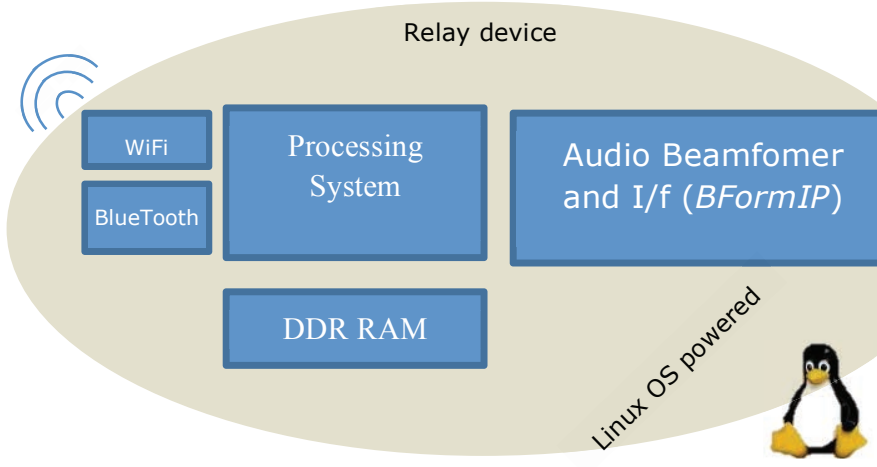

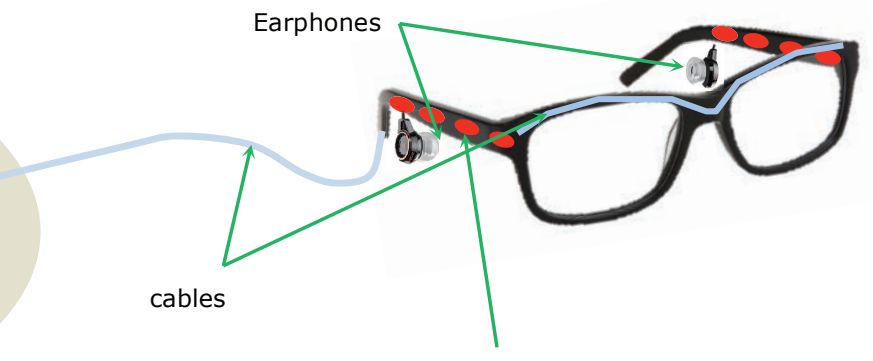

Digital MEMS Microphone

Figure 1 Block diagram of the Glassense system

is at least $5 \mathrm{~dB}$. For the considered application the choice of an end-fire array offers two main advantages respect to a broadband array. Firstly, an end-fire array inherently allows avoiding a beam pattern with grating lobes, i.e. a replicas of the main lobe, steered at the opposite of the direction of interest. Secondly, the geometry of the end-fire solution let to place one array for each head side, permitting to generate a more natural binaural signal.

\section{The Glassense System}

The Glassense system, depicted in Figure 1, is composed by 1) two superdirective microphone arrays mounted on the left and right frames of a pair of glasses 2) a relay device used to acquire and process audio signals 3 ) two earphones sending the processed signals to the ears. The microphone arrays are each made with 4 digital MEMs microphones. The relay device includes s a Xilinx Zedboard [13], a board containing everything necessary to create a Linux OS- based design. The Zedboard uses an ARM processing system and a programmable logic (PL) to create powerful and flexible designs.

\section{Superdirective Microphone array}

A filter-and-sum beamforming has been used to design an end-fire linear microphone array [14]. The array has 4 equally spaced elements over $0.12 \mathrm{~m}$ and each one feeds a FIR filter (i.e. a digital filter) of order 127 . The FIR filters working frequency band ranges from $400 \mathrm{~Hz}$ to $4 \mathrm{kHz}$, i.e. more than 3 octaves, and the frequency sampling is $16 \mathrm{kHz}$. The adopted technique allows attaining a frequency-invariant beam pattern with DI higher than an uniform linear array, i.e. to obtain superdirectivity. Further this technique is able to synthesize a beamformer robust to array imperfections [14], as errors in microphones response and position. In Figure 3 it is shown a polar representation of the nominal Beam Power Pattern (BPP), measured in dB, at $500 \mathrm{~Hz}, 1 \mathrm{kHz}$ and $2 \mathrm{kHz}$. As one notes the BPP has the 


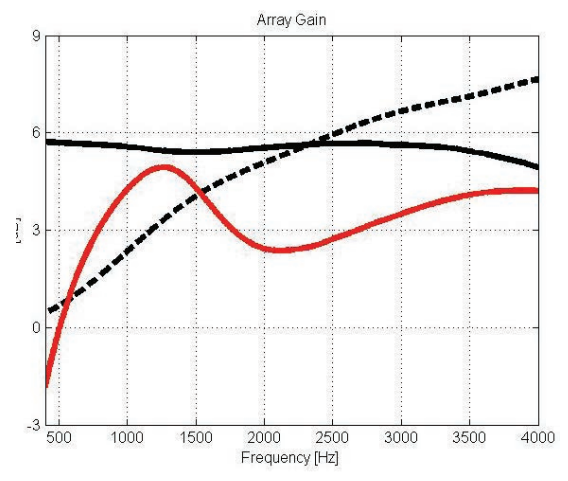

Figure 2 Directivity (black line) and white-noise gain (red line) of the end-fire array. The directivity of an uniformly weighted linear array (dashed black line) is shown for comparison
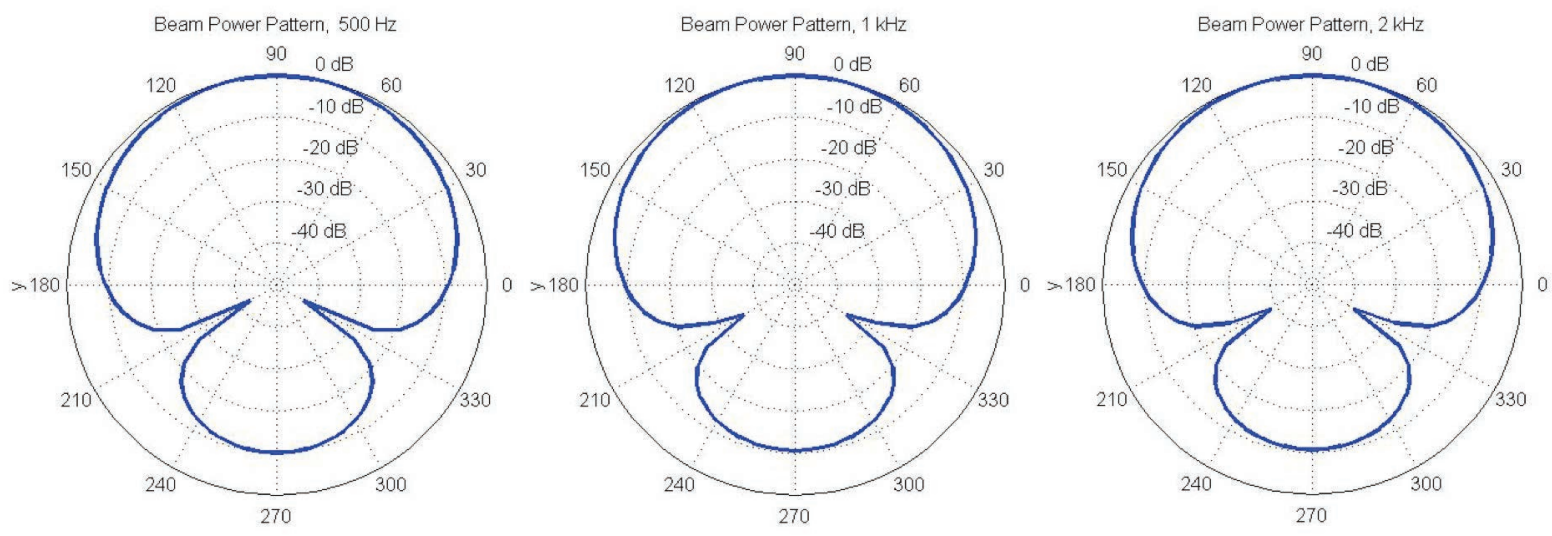

Figure 3 Beam Power Pattern at $500 \mathrm{~Hz}$ (left), 1 kHz (middle) and 2 kHz (right).

main lobe steered at $90 \mathrm{deg}$, with aperture (i.e., $-3 \mathrm{~dB}$ respect to the steering direction) of about $60 \mathrm{deg}$, and a single sidelobe at the opposite end-fire, i.e. $270 \mathrm{deg}$, about $10 \mathrm{~dB}$ lower. In Figure 2is plotted the DI value versus frequency for the same array. The designed array is superdirective for frequencies up to $2.3 \mathrm{kHz}$ (i.e. more than 2 octaves) with a mean DI value of $5.51 \mathrm{~dB}$ over the whole frequency band. Moreover, in Figure 2 is shown also a measure of the array robustness, i.e. the white-noise gain (WNG). A WNG level higher than $0 \mathrm{~dB}$ assure a positive robustness against realization imperfections. The same array design and beamformer structure has been used for the microphone array pair.

\section{Hardware architecture}

We developed a fully programmable design block inside the PL, called BformIP (see Figure 4), able to perform the superdirective beamforming for both left and right arrays without keeping busy the CPU.
The features of the beamformer are programmable, by accessing and configuring the BformIP internal registers. Therefore, the filter characteristics can be adapted to the end user preferences. The BformIp is connected to the processing system and then to the CPU through two different bus

- a bus used for setting the BformIP and

- $\quad$ another bus used for transferring the data read from microphones into the DDR RAM memory in a DMA (Direct Memory Access) master way port

Calibration of the single microphone gain is possible by adjusting a gain factor (see G0L, G1L blocks in Figure 4). The calibrated signal is then band-passed and processed with a FIR filter. It is also possible to access each of the eight single microphones for other needs. The left and right output of the beamformer are then fed to a codec via a stereo I2S protocol, then delivered 
either to the earphones or to the acoustic prosthetic.

This solution lets the beamformed signal to be delivered to the hearing system in real time and also to the operating system, which can redirect it to local applications or in the cloud. The BformIP can also mix the two beamformed audio channels with a synthesized audio coming from the auxiliary I2S interface, so that additional audio feedback from a smartphone/tablet (alerts and phone calls) can be superimposed to the output of the prosthesis.

\section{Software architecture}

On the software side, a kernel module containing a driver able to initialize the BformIP device at boot and to modify its configuration at runtime has been developed. During the initialization, the driver sets all the necessary configuration parameters: sampling frequency, calibration gains, microphones gains, bandSingle non-beamformed signals from microphones can also be accessed. As depicted in the Figure 4, the device transfers the audio samples to the operating system in the form of a four-channel raw audio stream containing left/right beamformed and left/right notbeamformed signal. The glassense driver lets the userspace applications to acquire all the signals in mono or stereo format and to play, record or stream them over the network. A last piece of software, the Glassense Config Tool, allows run-time read and edit of the configuration properties. Importantly, the system is versatile enough to load different pools of FIR filters, therefore spatial filtering of directions other that those in front of the user can be modulated according to the user needs.

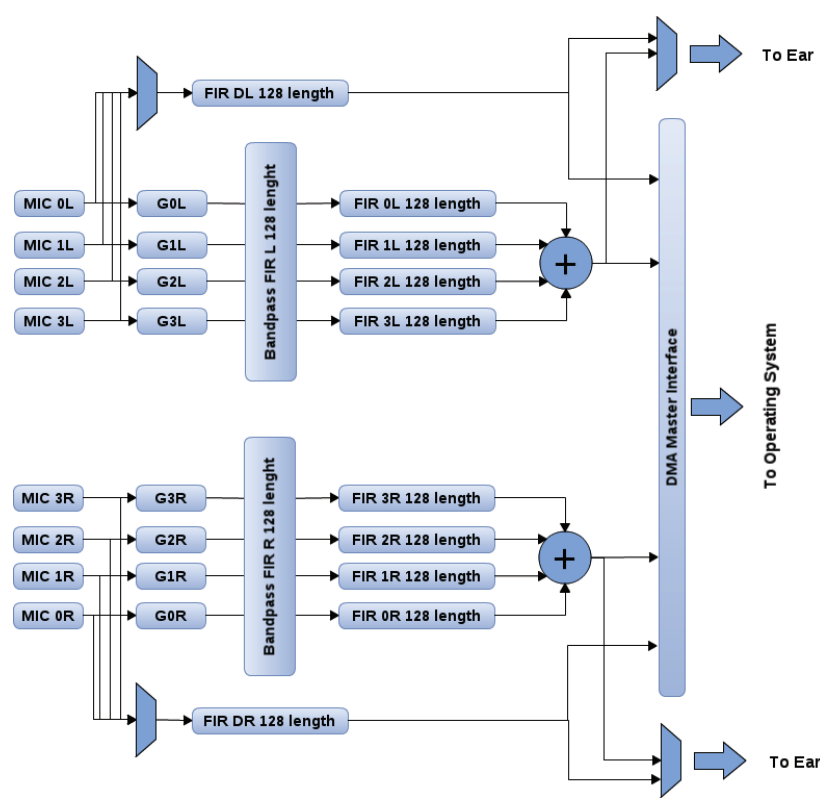

Figure 4 BForm IP block diagram

\section{Discussion}

We have proposed and built a system to potentially enhance speech intelligibility in hearing impaired individuals. A typical use case, planned with this device, can leverage on standard tests based on the percentage of understood sentences. The Glassense system can already be used as it is in such use case. Possible modifications depending on the use case performance concern the signal processing algorithms, therefore the systems appears to be very versatile.

Future hardware modifications regard ergonomics constraints only, e.g power consumption and weight, but the system is designed to allow easy miniaturization of most of its parts. 


\section{Acknowledgments}

This work is partly supported by the Ligurian PAR-FAS grant Glassense (CUP G35C13001360001) and partly by the EU FP7 grant BLINDPAD (grant number 611621).

\section{References}

1. Bronkhorst AW. The effect of head-induced interaural time and level differences on speech intelligibility in noise. J Acoust Soc Am. 1988;83 1508. doi: $10.1121 / 1.395906$

2. Wouters J, Klasen TJ, Moonen M. Distortion of interaural time cues by directional noise reduction systems in modern digital hearing aids, Katholieke Universiteit Leuven KBelgium. Signal Processing. 2005; 57-60.

3. Feuerstein JF. Ear and hearing. In: Monaural versus Binaural Hearing: Ease of Listening, Word Recognition, and Attentional Effort. 1992.

4. Kondo HM, Pressnitzer D, Toshima I, Kashino M. Effects of self-motion on auditory scene analysis. Proc Natl Acad Sci U S A. 2012;109: 6775-80. doi:10.1073/pnas.1112852109

5. Brimijoin WO, McShefferty D, Akeroyd MA. Auditory and visual orienting responses in listeners with and without hearing-impairment. J Acoust Soc Am. 2010;127: 3678-88. doi:10.1121/1.3409488

6. Van den Bogaert T, Doclo S, Wouters J, Moonen M Speech enhancement with multichannel Wiener filter techniques in multimicrophone binaural hearing aids. J Acoust Soc Am.; 2009;125: 36071. doi:10.1121/1.3023069
7. Neher T. Relating hearing loss and executive functions to hearing aid users' preference for, and speech recognition with, different combinations of binaural noise reduction and microphone directionality. Front Neurosci. 2014;8: 391. doi: $10.3389 /$ fnins. 2014.00391

8. Walden BE, Surr RK, Grant KW, Van Summers W, Cord MT, Dyrlund O. Effect of Signal-to-Noise Ratio on Directional Microphone Benefit and Preference. J Am Acad Audiol.2005;16: 662-676.

doi: $10.3766 /$ jaaa.16.9.4

9. Erin M. TAP, Elizabeth A, Ricketts. Potential Benefits and Limitations of Three Types of Directional Processing in Hearing Aids. Ear Hear. 2014; 35: 339-352.

doi:0.1097/AUD.0000000000000004

10. Zhang $X$, Huang J, Song $E$, Liu $H$, Li B, Yuan $X$. Design of small MEMS microphone array systems for direction finding of outdoors moving vehicles. Sensors (Basel). Multidisciplinary Digital Publishing Institute; 2014;14: 4384-98.

doi:10.3390/s140304384

11. Hoffman MW. Robust adaptive microphone array processing for hearing aids: Realistic speech enhancement. J Acoust Soc Am.; 1994;96: 759. doi: $10.1121 / 1.410313$

12. Dillon H. Hearing aids. 2001;

13. Zedboard Hardware User's Guide, Version 2.2, Avnet. 2014.

14. Crocco M, Trucco A. The synthesis of robust broadband beamformers for equally-spaced linear arrays. J Acoust Soc Am. 2010;128: 691-701. doi:10.1121/1.3455852 